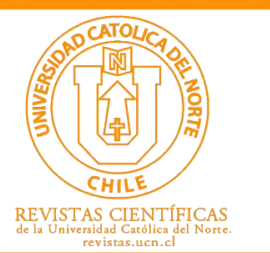

\title{
Distance and eccentricity based polynomials and indices of $m$-level Wheel graph
}

\author{
Murat Cancan $^{1}$ [D orcid.org/0000-0002-8606-2274

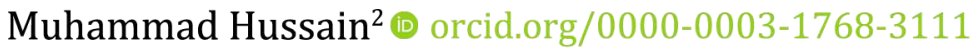 \\ Haseeb Ahmad ${ }^{3}$ (ib orcid.org/0000-0003-2015-371X \\ ${ }^{1}$ Van Yznc Yil University, Faculty of Education, Van, Turkey. \\ m_cencen@yahoo.com \\ ${ }^{2}$ COMSATS University Islamabad, Dept. of Mathematics, Lahore, Pakistan. \\ mhmaths@gmail.com \\ ${ }^{3}$ Lahore Leads University, Dept. of Mathematics, Lahore, Pakistan. \\ haseeb.ahmad@leads.edu.pk \\ Received: February 2020 | Accepted: May 2020
}

\section{Abstract:}

Distance and degree based topological polynomial and indices of molecular graphs have various applications in chemistry, computer networking and pharmacy. In this paper, we give hosoya polynomial, Harary polynomial, Schultz polynomial, modified Schultz polynomial, eccentric connectivity polynomial, modified Wiener index, modified hyper Wiener index, generalized Harary index, multiplicative Wiener index, Schultz index, modified Schultz index, eccentric connectivity index of generalized wheel networks $W_{n, m}$. We also give pictorial representation of computed topological polynomials and indices on the involved parameters $m$ and $n$.

Keywords: Hosoya polynomial; Harary polynomial; Schultz polynomial; Eccentric connectivity polynomial; Wiener index; Schultz index; Eccentric connectivity index; $m$-level wheel graph.

MSC (2020): 05C09, 05C12.

\section{Cite this article as (IEEE citation style):}

M. Cancan, M. Hussain, and H. Ahmad, "Distance and eccentricity based polynomials and indices of $m$-level Wheel graph", Proyecciones (Antofagasta, On line), vol. 39, no. 4, pp. 869-885, Aug. 2020, doi: 10.22199/issn.0717-6279-2020-04-0054

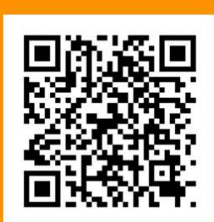

Article copyright: (C) 2020 Murat Cancan, Muhammad Hussain and Haseeb Ahmad. This is an open access article distributed under the terms of the Creative Commons License, which permits unrestricted use and distribution provided the original author and source are credited. 


\section{Introduction}

A simple graph $G=(V, E)$ is a finite nonempty set $V(G)$ of objects called vertices together with a (possibly empty) set $E(G)$ of unordered pairs of distinct vertices of $G$ called edges.

A topological index is a numerical quantity derived in an unambiguous manner from the structure graph of a molecule. As a graph structural invariant, i.e. it does not depend on the labeling or the pictorial representation of a graph. Various topological indices usually reflect molecular size and shape.

The Hosoya polynomial of a graph is a generating function about distance distributing, introduced by Haruo Hosoya in 1998 [17] and for a connected graph $G$ is defined as:

$$
H(G, x)=\frac{1}{2} \sum_{v \in V(G)} \sum_{u \in V(G)} x^{d(v, u)},
$$

where $d(u, v)$ denotes the distance between vertices $u$ and $v$.

The Hosoya polynomial has many chemical applications [16] especially Wiener index can be directly obtained from the polynomial and studied extensively $[9,10,26]$.

The main advantage of the Hosoya polynomial is that it contains a wealth of information about distance based graph invariants. For instance, knowing the Hosoya polynomial of a graph, it is straight forward to determine the famous Wiener index of a graph as the first derivative of the polynomial. The Wiener index of a connected graph $G$ is denoted by $W(G)$ and defined as the sum of distances between all pairs of vertices in $\mathrm{G}$ i.e.

$$
W(G)=\frac{1}{2} \sum_{v \in V(G)} \sum_{u \in V(G)} d(u, v) .
$$

The modified Wiener index of a connected graph $G$ is denoted by $W_{\lambda}(G)$ and defined as the sum of $\lambda$ power distances between all pairs of vertices in $G$, where $\lambda=\{1,2,3,4, \ldots\}$. It can be formulated as

$$
\mathrm{W}_{\lambda}(G)=\frac{1}{2} \sum_{v \in V(G)} \sum_{u \in V(G)} d(u, v)^{\lambda}
$$

The Wiener index was introduced by Harold Wiener in 1947 to study the boiling points of paraffin [31]. It plays an important role in the so-called inverse structure-property relationship problems [11]. For more details about 
this topological polynomial and index, please see the paper series and the references therein $[3,6,15,23,27,30]$. Note that the first derivative of the Hosoya polynomial at $x=1$ is equal to the Wiener index:

$$
W(G)=\left.\frac{\partial H(g)}{\partial x}\right|_{x=1}
$$

Hyper-Wiener index is another distance-based graph invariants used for predicting physico-chemical properties of organic compounds [20]. The Hyper-Wiener index was introduced by M. Randić [31] as:

$$
W W(G)=\frac{1}{2} \sum_{v \in V(G)} \sum_{u \in V(G)}\left(d(u, v)+d(u, v)^{2}\right) .
$$

Modified Hyper Wiener index of a connected graph $G$ is denoted by $W W_{\lambda}(G)$ and defined as:

where $\lambda=\{1,2,3,4, \ldots\}$

$\mathrm{WW}_{\lambda}(G)=\frac{1}{2} \sum_{v \in V(G)} \sum_{u \in V(G)}\left(d(u, v)^{\lambda}+d(u, v)^{2 \lambda}\right)$.

The Schultz index of a molecular graph $G$ was introduced by Schultz [22] in 1989 for characterizing alkanes by an integer as follow:

$$
S c(G)=\frac{1}{2} \sum_{v \in V(G)} \sum_{u \in V(G)}\left(d_{u}+d_{v}\right) d(u, v)
$$

The modified Schultz index of a graph $G$ was introduced by S. Klavar and I. Gutman in 1996 as follow [18]:

$$
S c^{*}(G)=\frac{1}{2} \sum_{v \in V(G)} \sum_{u \in V(G)}\left(d_{u} d_{v}\right) d(u, v) .
$$

The Schultz and modified Schultz polynomials of $G$ are defined as [4]:

$$
\begin{aligned}
& S c(G)=\frac{1}{2} \sum_{v \in V(G)} \sum_{u \in V(G)}\left(d_{u}+d_{v}\right) x^{d(u, v)} \\
& S c^{*}(G)=\frac{1}{2} \sum_{v \in V(G)} \sum_{u \in V(G)}\left(d_{u} d_{v}\right) x^{d(u, v)}
\end{aligned}
$$

The Harary polynomial of a connected graph $G$ is denoted by $h(G, x)$ and defined as

$$
h(G, x)=\frac{1}{2} \sum_{v \in V(G)} \sum_{u \in V(G)} \frac{1}{d(v, u)} x^{d(v, u)},
$$


The generalized Harary index of a connected graph $G$ is denoted by $h_{t}(G)$ and defined as

$$
h_{t}(G)=\sum_{v \in V(G)} \sum_{u \in V(G)} \frac{1}{d(v, u)+t}
$$

The multiplicative Wiener index of a connected graph $G$ is denoted by $\pi(G)$ and defined as

$\pi(G)=\prod_{u, v \subseteq V(G)} d(u, v)$

The eccentricity $e c(v)$ of vertex $v \in V(G)$ is the maximum distance between $v$ and any other vertex in $G$. Then the eccentric connectivity index(ECI) of $G$ was introduced by Sharma and Madan [24] as

$$
\xi(G)=\sum_{v \in V(G)} \sum_{u \in V(G)} d(v, u) \varepsilon(v)
$$

As the extension of eccentric connectivity index, eccentric connectivity polynomial was introduced by Ashraf and Jalali [5] which was denoted as

$$
\xi(G, x)=\sum_{v \in V(G)} \sum_{u \in V(G)} d(v, u) x^{\varepsilon(v)}
$$

The wheel graph has been used in different areas such as the wireless sensor networks and the vulnerability of networks [7]. The wheel graph has many good properties. From the standpoint of the hub vertex, all elements, including vertices and edges, are in its one-hop neighborhood, which indicates that the wheel structure is fully included in the neighborhood graph of the hub vertex. Wheel and related graphs are extensively studied recently. In [28], the authors computed partition dimension and connected partition dimension of wheel graphs. In [29], the authors gave an algorithm to compute average lower two-domination number and also computed this number for some wheel related graphs. In [25], authors computed the metric dimension of generalised wheel. For more details see $[2,19,21,30]$. For detailed applications of our results we refer $[1,8,12,13,14]$ and references therein.

In this report, we study Hosoya polynomial, Harary polynomial, Schultz polynomial, modified Schultz polynomial, eccentric connectivity polynomial, modified Wiener index, modified hyper Wiener index, generalized harary index, multiplicative Wiener index, Schultz index, modified Schultz index and eccentric connectivity index of multi-level wheel graph. 


\section{Main Results}

Definition 1 ( $m$-level wheel graph). Generalized wheel graph, also known as multi-level wheel network, $W_{m, n}$. An multi-level wheel network $W_{m, n}$ is a network obtained from $m$-copies of cycles $C_{n}$ and one copy of vertex $v$, such that all vertices of every copy of $C_{n}$ are adjacent to $v$. Thus, $W_{m, n}$ has $m n+1$ vertices, i.e. the center and $n$-rim vertices, and has diameter 2. is shown in figure 1 .

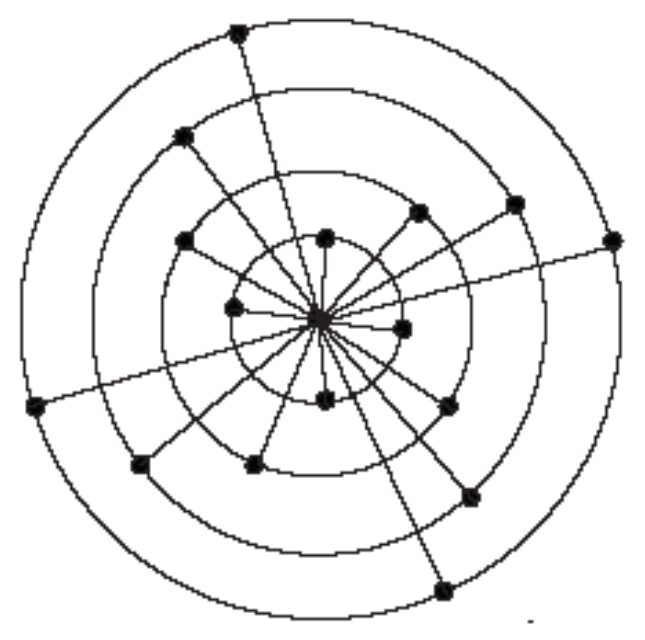

Figure 2.1: $W_{4,4}$.

Lemma 1. The graph $W_{m, n}$ having $m n+1$ vertices and $2 m n$ edges.

Theorem 2.1. If $W_{m, n}$ is the $m$-wheel graph $m \geq 2, n \geq 3$, then

$$
H\left(W_{m, n} ; x\right)=m n+1+2 m n x+\left(\frac{m^{2} n^{2}}{2}-\frac{3 m n}{2}\right) x^{2}
$$

Proof. As first term of Hosoya polynomial is number of vertices which can be easily computed by Figure $2.1\left|V\left(W_{n}^{m}\right)\right|=m n+1$. For the second term of Hosoya polynomial is number of edges, central vertex having $\mathrm{nm}$ degree and other $n m$ vertex having 3 degree.

$$
\left|E\left(W_{n}^{m}\right)\right|=\frac{(m n+3 m n) m}{2}=2 m n
$$


For the third term of Hosoya polynomial of $W_{n}^{m}$, we see that the central central vertex having only 1 -edge paths between all other vertices so remaining $m n$ vertices having 2 -edge paths between all other vertices except central vertex i.e $m n$. So first $n$ vertices of $C_{n}$ of $W_{m, n}$ having $2-$ edge paths with all other $(m-1)$ copies of $C_{n}$ having $n$ vertices, that is $n^{2}(m-1)$. Similarly second $n^{2}$ join with remaining $(m-2)$ copies, i.e $n^{2}(m-2)$ and so on.

$$
\begin{aligned}
= & (\mathrm{n})^{2}(m-1)+(n-1)^{2}(m-2)+(n-1)^{2}(m-3)+\ldots+(n-1)^{2} 2+(n-1)^{2} \\
& =(n-1)^{2}[(m-1)+(m-2)+(m-3)+\ldots+2+1] \\
& =\frac{n^{2} m(m-1)}{2}
\end{aligned}
$$

as wheel graph having $\frac{n^{2}}{2}-\frac{3 n}{2}, 2-$ edge paths and generalized wheel graph having $m$-wheels so $m$ times 2 -edge paths i.e $m\left(\frac{n^{2}}{2}-\frac{3 n}{2}\right)$ now, the coefficient of the third term of Hosoya polynomial will be

$$
\begin{aligned}
& =\frac{n^{2} m(m-1)}{2}+m\left(\frac{n^{2}}{2}-\frac{3 n}{2}\right) \\
& =\frac{m^{2} n^{2}}{2}-\frac{3 m n}{2}
\end{aligned}
$$

Here, by what have been mentioned above, we have following computations for the Hosoya polynomial of $m$-wheel graph $W_{n}^{m}$.

$$
H\left(W_{m, n} ; x\right)=m n+1+2 m n x+\left(\frac{m^{2} n^{2}}{2}-\frac{3 m n}{2}\right) x^{2}
$$

\begin{tabular}{|c|c|c|c|}
\hline \multicolumn{4}{|c|}{ Table 1. Hosoya polynomial of $\left(W_{m, n}\right)$} \\
\hline Distances & 0 & 1 & 2 \\
\hline Terms of Hosoya polynomial & $m n+1$ & $2 m n$ & $\frac{m^{2} n^{2}}{2}-\frac{3 m n}{2}$ \\
\hline
\end{tabular}

Theorem 2.2. For the $m$-wheel graph $W_{m, n}$, the harary polynomial is

$$
h\left(W_{m, n} ; x\right)=2 m n x+\left(\frac{m^{2} n^{2}}{4}-\frac{3 m n}{4}\right) x^{2}
$$


Proof. From table 1

$$
\begin{aligned}
h\left(W_{m, n}, x\right) & =\sum_{v \in V\left(W_{m, n}\right)} \sum_{u \in V\left(W_{m, n}\right)} \frac{1}{d(u, v)} x^{d(u, v)} \\
& =\sum_{v \in V\left(W_{m, n}\right)} \sum_{u \in V\left(W_{m, n}\right)} \frac{1}{1} x^{1}+\sum_{v \in V\left(W_{m, n}\right)} \sum_{u \in V\left(W_{m, n}\right)} \frac{1}{2} x^{2} \\
h\left(W_{m, n} ; x\right) & =2 m n x+\left(\frac{m^{2} n^{2}}{4}-\frac{3 m n}{4}\right) x^{2}
\end{aligned}
$$

Theorem 2.3. For the $m$-wheel graph $W_{m, n}$, we have

1. $W_{\lambda}\left(W_{m, n}\right)=2 m n+\left(m^{2} n^{2}-3 m n\right) 2^{\lambda-1}$

2. $W W_{\lambda}\left(W_{m, n}\right)=4 m n+\left(m^{2} n^{2}-3 m n\right)\left(2^{\lambda-1}+2^{2 \lambda-1}\right)$

3. $h_{t}\left(W_{m, n}\right)=\frac{2 m n}{(1+t)}+\frac{m^{2} n^{2}-3 m n}{2(2+t)}$

4. $\pi\left(W_{m, n}\right)=1^{2 m n} \times 2^{\left(\frac{m^{2} n^{2}}{2}-\frac{3 m n}{2}\right)}$

Proof. As modified Wiener index is

$$
\begin{aligned}
W_{\lambda}\left(W_{m, n}\right) & =\frac{1}{2} \sum_{v \in V\left(W_{m, n}\right)} \sum_{u \in V\left(W_{m, n}\right)} d(u, v)^{\lambda} \\
& =\frac{1}{2} \sum_{v \in V\left(W_{m, n}\right)} \sum_{u \in V\left(W_{m, n}\right)}(1)^{\lambda}+\frac{1}{2} \sum_{v \in V\left(W_{m, n}\right)} \sum_{u \in V\left(W_{m, n}\right)}(2)^{\lambda} \\
& =2 m n+\left(m^{2} n^{2}-3 m n\right) 2^{\lambda-1}
\end{aligned}
$$

In Table 2 modified Wiener index of $m$-wheel graphs for $n=9$ and different values of $m$ and $\lambda$.

\begin{tabular}{|c|c|c|c|c|c|c|}
\hline \multicolumn{6}{|c|}{ Table 2. Modified Wiener index of $\left(W_{\lambda}\left(W_{m, 9}\right)\right.$} \\
\hline$\lambda \backslash m$ & 1 & 2 & 3 & 4 & 5 & 6 \\
\hline 1 & 72 & 306 & 702 & 1260 & 1980 & 2862 \\
\hline 2 & 126 & 576 & 1350 & 2448 & 38707 & 5616 \\
\hline 3 & 234 & 1116 & 2646 & 4824 & 7650 & 11124 \\
\hline 4 & 450 & 2196 & 5238 & 9576 & 15210 & 22140 \\
\hline 5 & 882 & 4356 & 10422 & 19080 & 30330 & 44172 \\
\hline 6 & 1746 & 8676 & 20790 & 38088 & 60570 & 88236 \\
\hline
\end{tabular}


For modified Hyper Wiener index

$$
\begin{aligned}
W W_{\lambda}\left(W_{m, n}\right) & =\frac{1}{2}\left(\sum_{v \in V\left(W_{m, n}\right)} \sum_{u \in V\left(W_{m, n}\right)} d(u, v)^{\lambda}+\sum_{v \in V\left(W_{m, n}\right)} \sum_{u \in V\left(W_{m, n}\right)} d(u, v)^{2 \lambda}\right) . \\
& =W_{\lambda}\left(W_{m, n}\right)+\frac{1}{2} \sum_{v \in V\left(W_{m, n}\right)} \sum_{u \in V\left(W_{m, n}\right)} d(u, v)^{2 \lambda} . \\
& =W_{\lambda}\left(W_{m, n}\right)+\frac{1}{2} \sum_{v \in V\left(W_{m, n}\right)} \sum_{u \in V\left(W_{m, n}\right)}(1)^{2 \lambda} \\
& +\frac{1}{2} \sum_{v \in V\left(W_{m, n}\right)} \sum_{u \in V\left(W_{m, n}\right)}(2)^{2 \lambda} \\
& =\frac{1}{2} \sum_{v \in V\left(W_{m, n}\right)} \sum_{u \in V\left(W_{m, n}\right)}\left(1^{\lambda}+1^{2 \lambda}\right) \\
& +\frac{1}{2} \sum_{v \in V\left(W_{m, n}\right)} \sum_{u \in V\left(W_{m, n}\right)}\left(2^{\lambda}+2^{2 \lambda}\right) \\
W W_{\lambda}\left(W_{m, n}\right) & =4 m n+\left(m^{2} n^{2}-3 m n\right)\left(2^{\lambda-1}+2^{2 \lambda-1}\right)
\end{aligned}
$$

In table 3 modified Hyper-Wiener index of $m$-wheel graphs for $n=9$ and different values of $m$ and $\lambda$.

\begin{tabular}{|c|c|c|c|c|c|c|}
\hline \multicolumn{6}{|c|}{ Table 3. Modified Hyper Wiener index of $\left(W W_{\lambda}\left(W_{m, 9}\right)\right.$} \\
\hline$\lambda \backslash m$ & 1 & 2 & 3 & 4 & 5 & 6 \\
\hline 1 & 198 & 882 & 2052 & 3708 & 5850 & 8478 \\
\hline 2 & 576 & 2772 & 6588 & 12024 & 19080 & 27756 \\
height3 & 1980 & 9792 & 23436 & 42912 & 68220 & 99360 \\
\hline 4 & 7380 & 36792 & 88236 & 161712 & 257220 & 374760 \\
\hline 5 & 28548 & 142632 & 342252 & 627408 & 998100 & 1454328 \\
\hline 6 & 112356 & 561672 & 1347948 & 2471184 & 3931380 & 5728536 \\
\hline
\end{tabular}

For generalized harary index

$$
\begin{aligned}
h_{t}\left(W_{m, n}\right) & =\frac{1}{2} \sum_{v \in V\left(W_{m, n}\right)} \sum_{u \in V\left(W_{m, n}\right)} \frac{1}{d(u, v)+t} . \\
& =\frac{1}{2} \sum_{v \in V(G)} \sum_{u \in V(G)} \frac{1}{1+t}+\frac{1}{2} \sum_{v \in V\left(W_{m, n}\right)} \sum_{u \in V\left(W_{m, n}\right)} \frac{1}{2+t} \\
& =\frac{2 m n}{(1+t)}+\frac{m^{2} n^{2}-3 m n}{2(2+t)}
\end{aligned}
$$


In table 4 generalized Harary index of $m$-wheel graphs for $n=9$ and different values of $m$ and $t$.

\begin{tabular}{|c|c|c|c|c|c|c|}
\hline \multicolumn{7}{|c|}{ Table 4. generalized Harary index of $\left.\left(H_{t} W_{m, 9}\right)\right)$} \\
\hline$t \backslash m$ & 1 & 2 & 3 & 4 & 5 & 6 \\
\hline 0 & $63 / 2$ & $207 / 2$ & 216 & 369 & $1125 / 2$ & $1593 / 2$ \\
height1 & 18 & 63 & 135 & 234 & 360 & 513 \\
height2 & $51 / 4$ & $183 / 4$ & 99 & $345 / 2$ & $1065 / 4$ & $1521 / 4$ \\
height3 & $99 / 10$ & 36 & $783 / 10$ & $684 / 5$ & $423 / 2$ & $1512 / 5$ \\
\hline 4 & $81 / 10$ & $297 / 10$ & $324 / 5$ & $567 / 5$ & $351 / 2$ & $2511 / 10$ \\
\hline 5 & $48 / 7$ & $177 / 7$ & $387 / 7$ & $678 / 7$ & 150 & $1503 / 7$ \\
\hline 6 & $333 / 56$ & $1233 / 56$ & $675 / 14$ & $2367 / 28$ & $7335 / 56$ & $10503 / 56$ \\
\hline
\end{tabular}

For Multiplicative Wiener index

$$
\begin{aligned}
\pi(G) & =\prod_{u, v \subseteq V\left(W_{m, n}\right)} d(u, v) \\
& =\prod_{u, v \subseteq V\left(W_{m, n}\right)}(1) \times \prod_{u, v \subseteq V\left(W_{m, n}\right)}(2) \\
& =1^{2 m n} \times 2^{\left(\frac{m^{2} n^{2}}{2}-\frac{3 m n}{2}\right)}
\end{aligned}
$$

Corollary 2.4. The Wiener index of $m$-wheel graph $W_{m, n},(m \geq 2, n \geq 3)$ is

$$
W_{1}\left(W_{m, n}\right)=m^{2} n^{2}-m n .
$$

Corollary 2.5. The Hyper-Wiener index of $m$-wheel graph $W_{m, n},(m \geq$ $2, n \geq 3)$ is

$$
W W_{1}\left(W_{m, n}\right)=3 m^{2} n^{2}-5 m n
$$

Corollary 2.6. The Harary index of $m$-wheel graph $W_{m, n},(m \geq 2, n \geq 3)$ is

$$
h_{0}\left(W_{m, n}\right)=\frac{m^{2} n^{2}+5 m n}{4} .
$$

Theorem 2.7. If $W_{m, n}$ is the $m$-wheel graph $m \geq 2, n \geq 3$, then

$$
\begin{gathered}
S c\left(W_{m, n} ; x\right)=\left(9 m n+m^{2} n^{2}\right) x+\left(3 m^{2} n^{2}-9 m n\right) x^{2} \\
S c^{*}\left(W_{m, n} ; x\right)=\left(9 m n+3 m^{2} n^{2}\right) x+\left(\frac{9 m^{2} n^{2}}{2}-\frac{27 m n}{2}\right) x^{2} .
\end{gathered}
$$


Proof. As $d(u, v) \in\{1,2\}$ and $d_{u}=i, d_{v}=j$. To prove these results we need to compute $i$-th paths and $i$-th term of Schultz and modified Schultz polynomials as commuted in table 5 . The number of $i-$ th component of Schultz polynomial is sum of degrees of corresponding vertices multiplied by total number of $i$-th paths and the number of $i$-th component of modified Schultz polynomial is product of degrees of corresponding vertices multiplied by total number of $i$-th paths.

\begin{tabular}{|l|l|l|l|l|}
\hline \multicolumn{5}{|c|}{ Table 5. The number of all distinct types of 1 and 2-edge-paths } \\
\hline $\begin{array}{l}i-t h \text { edge } \\
\text { paths }\end{array}$ & $d_{u}, d_{v}$ & $\begin{array}{l}\text { Number of } \\
i-\text { th paths }\end{array}$ & $\begin{array}{l}\text { terms of } \\
\text { Schultz } \\
\text { polynomial }\end{array}$ & $\begin{array}{l}\text { terms of Modified } \\
\text { Schultz } \\
\text { polynomial }\end{array}$ \\
\hline 1 & 3,3 & $m n$ & $6 m n$ & $9 m n$ \\
\hline 1 & $3, m$ & $m n$ & $(3+m n) m n$ & $3 m^{2} n^{2}$ \\
\hline 2 & 3,3 & $\frac{m^{2} n^{2}}{2}-\frac{3 m n}{2}$ & $3 m^{2} n^{2}-9 m n$ & $\frac{9 m^{2} n^{2}}{2}-\frac{27 m n}{2}$ \\
\hline
\end{tabular}

Therefore, the Schultz, Modified Schultz polynomials of the $m$-level wheel graph $W_{m, n}$ are equal to:

$$
\begin{aligned}
S c\left(W_{m, n}, x\right) & =\frac{1}{2} \sum_{v \in V(G)}\left(d_{u}+d_{v}\right) x^{d(u, v)} \\
& =((6) m n+(3+m n) m n) x+(6)\left(\frac{m^{2} n^{2}}{2}-\frac{3 m n}{2}\right) x^{2} \\
& =\left(9 m n+m^{2} n^{2}\right) x+\left(3 m^{2} n^{2}-9 m n\right) x^{2} \\
S c^{*}\left(W_{m, n}, x\right) & =\frac{1}{2} \sum_{v \in V(G)}\left(d_{u} d_{v}\right) x^{d(u, v)} \\
& =((9) m n+(3 m n) m n) x+(9)\left(\frac{m^{2} n^{2}}{2}-\frac{3 m n}{2}\right) x^{2} \\
& =\left(9 m n+3 m^{2} n^{2}\right) x+\left(\frac{9 m^{2} n^{2}}{2}-\frac{27 m n}{2}\right) x^{2}
\end{aligned}
$$


Theorem 2.8. If $W_{m, n}$ is the $m$-wheel graph $m \geq 2, n \geq 3$, then

$$
\begin{gathered}
S c\left(W_{m, n}\right)=7 m^{2} n^{2}-9 m n \\
S c^{*}\left(W_{m, n}\right)=12 m^{2} n^{2}-18 m n .
\end{gathered}
$$

Proof. Consider the $m$-wheel graph $W_{m, n}$ from the definition of Schultz index and the Modified Schultz index of a graph $G$, we can see the Schultz and Modified Schultz indices of $G$, by the first derivative of their polynomials (evaluated at $\mathrm{x}=1$ ). So, by using above mentions results, we have following computations

$$
\begin{aligned}
S c\left(W_{m, n}\right) & =\left.\frac{\partial S c\left(W_{m, n}, x\right)}{\partial x}\right|_{x=1} \\
& =\frac{\partial}{\partial x}\left[\left(9 m n+m^{2} n^{2}\right) x+\left.\left(3 m^{2} n^{2}-9 m n\right) x^{2}\right|_{x=1}\right. \\
& =7 m^{2} n^{2}-9 m n \\
S c^{*}\left(W_{m, n}\right) & =\left.\frac{\partial S c^{*}\left(W_{m, n}, x\right)}{\partial x}\right|_{x=1} \\
& =\left.\frac{\partial}{\partial x}\left[\left(9 m n+3 m^{2} n^{2}\right) x+\left(\frac{9 m^{2} n^{2}}{2}-\frac{27 m n}{2}\right)\right]\right|_{x=1} \\
& =12 m^{2} n^{2}-18 m n
\end{aligned}
$$

Theorem 2.9. If $W_{m, n}$ is the $m$-wheel graph $m \geq 2, n \geq 3$, then

$$
\begin{aligned}
\xi\left(W_{m, n}, x\right) & =m n x+3 m n x^{2} \\
& =7 m n
\end{aligned}
$$

Proof. Consider the $m$-wheel graph $W_{m, n}$, this graph has a central vertex such as $u$, with $e c c(u)=1$ and other $m n$ vertices say $v_{i}$ having $\operatorname{ecc}\left(v_{i}\right)=2$ and degrees of $u$ and $v_{i}^{\prime} s$ are $m n$ and 3 respectively. By the definition eccentric connectivity polynomial, we have following computations 


$$
\begin{aligned}
\xi\left(W_{m, n}, x\right) & =\sum_{v \in V\left(W_{m, n}\right)} d(v, u) x^{\varepsilon(v)} \\
& =m n x+3 m n x^{2}
\end{aligned}
$$

the definition of eccentric connectivity index, we can see the eccentric connectivity index of $G$, by the first derivative of their polynomials (evaluated at $\mathrm{x}=1$ ). So, by using above mentions results, we have following computations

$$
\begin{aligned}
\xi\left(W_{m, n}\right) & =\left.\frac{\partial \xi\left(W_{m, n}, x\right)}{\partial x}\right|_{x=1} \\
& =\left.\frac{\partial}{\partial x}\left[m n x+3 m n x^{2}\right]\right|_{x=1} \\
& =7 m n
\end{aligned}
$$

\section{Graphical representation and concluding remarks}

In the current article, we computed distances based topological polynomials and indices of multi-level wheels, which are the extension of classical wheel graph. Figure 3.1 shows the comparison of Hosoya polynomial, Harary polynomial, Schultz polynomial, modified Schultz polynomial and eccentric connectivity polynomial with fixed value of $m$ and $n$ i.e $m=7, n=12$. Every polynomial is exponentially increasing but modified Schultz is faster and eccentric connectivity is slower. Figure 3.2 depict the mesh polting and comparison of Wiener index, Hyper Wiener index, Harary index, Schultz index, modified Schultz index and Eccentric connectivity index with $2 \geq$ $m \geq 50,3 \geq n \geq 50$. 


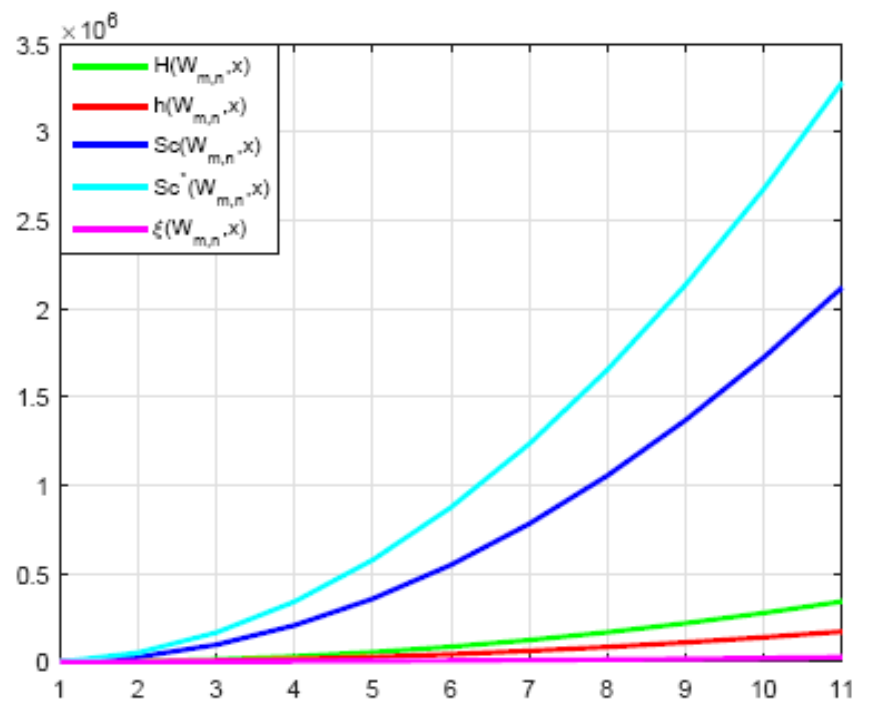

Figure 3.1: Polynomials of $W_{m, n}$ for $m=7, n=12$.

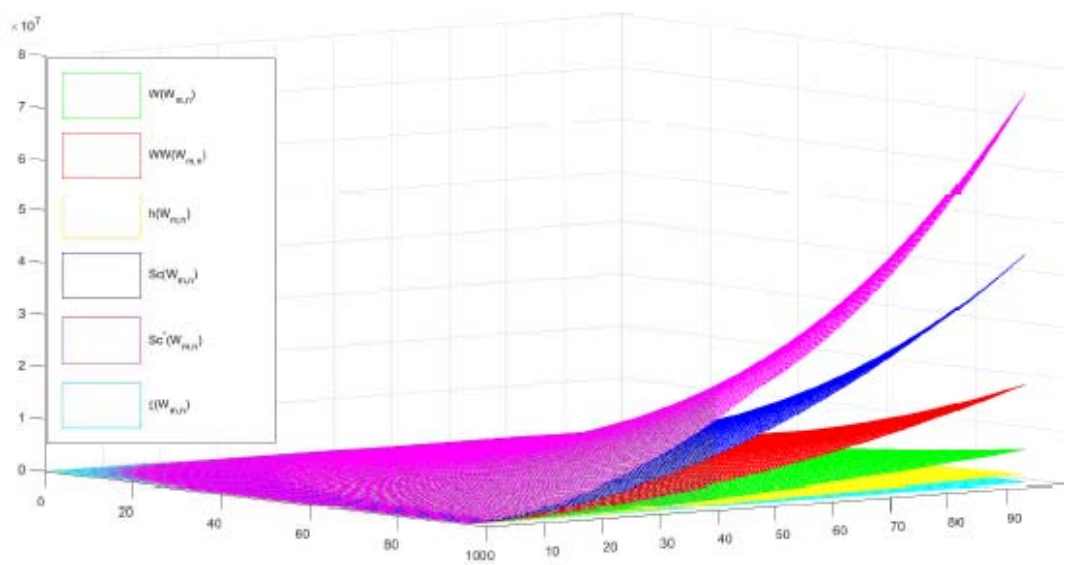

Figure 3.2: Indices of $W_{m, n}$ for $2 \geq m \geq 50,3 \geq n \geq 50$. 


\section{References}

[1] S. Ahmad, H. M. A. Siddiqui, A. Ali, M. R. Farahani, M. Imran, and I. N. Cangul, "On Wiener index and Wiener polarity index of some polyomino chains", Journal of discrete mathematical sciences and cryptography, vol. 22, no. 7, pp. 1151-1164, Oct. 2019, doi: 10.1080/09720529.2019.1688965

[2] A. Ali, W. Nazeer, M. Munir, and S. M. Kang, "M-polynomials and topological indices of zigzag and rhombic benzenoid systems", Open chemistry, vol. 16, no. 1, pp. 73-78, Feb. 2018, doi: 10.1515/chem2018-0010

[3] U. Ali, Y. Ahmad, and M. S. Sardar, "On 3-total edge product cordial labeling of tadpole, book and flower graphs", Open journal of mathematical sciences, vol. 4, no. 1 , pp. 48-55, Mar. 2020, doi: $10.30538 /$ oms 2020.0093

[4] Y. Alizadeh, A. Iranmanesh and S. Mirzaie, "Computing Schultz Polynomial, Schultz Index of C60 Fullerene By Gap Program", Digest journal of nanomaterials and biostructures, vol. 4, no. 1, pp. 7-10, 2009. [On line]. Available: https://bit.ly/3hRts8L

[5] A. R. Ashrafi, M. Ghorbani, and M. Jalali, "Eccentric connectivity polynomial of an infinite family of fullerenes", Optoelectronics and advanced materials - rapid communications, vol. 3, no. 8, pp. 823-826, Jul. 2009. [On line]. Available: https://bit.ly/3doNGTP

[6] F. Asif, Z. Zahid and S. Zafar, "Leap Zagreb and leap hyperZagreb indices of Jahangir and Jahangir derived graphs", Engineering and applied science letter, vol. 3, no. 2, pp. 1-8, 2020. [On line]. Available: https://bit.ly/2Bmb1YP

[7] A. Aytac and T. Turaci, "Vertex vulnerability parameter of gear graphs", International journal of foundations of computer science, vol. 22, no. 05, pp. 1187-1195, Aug. 2011, doi: $10.1142 / \mathrm{S} 0129054111008635$

[8] M. Cancan, S. Ediz, and M. R. Farahani, "On ve-degree atom-bond connectivity, sum-connectivity, geometric-arithmetic and harmonic indi- 
ces of copper oxide", Eurasian chemical communications, vol. 2, no. 5, pp. 641-645, Mar. 2020, doi: 10.33945/SAMI/ECC.2020.5.11

[9] G. G. Cash, "Relationship between the Hosoya polynomial and the hyper-Wiener index", Applied mathematics letters, vol. 15, no. 7, pp. 893-895, 2002, doi: 10.1016/S0893-9659(02)00059-9

[10] M. V. Diudea. "Hosoya polynomial in tori", MATCH communication in mathematical and in computer chemistry, vol. 45, pp. 109-122, 2002. [On line]. Available: https://bit.ly/3ehNKpr

[11] A. A. Dobrynin, R. Entringer, and I. Gutman, "Wiener index of trees: theory and applications", Acta applicandae mathematicae, vol. 66, no. 3, pp. 211-249, 2001, doi: 10.1023/A:1010767517079

[12] W. Gao and M. R. Farahani, "The hyper-zagreb index for an infinite family of nanostar dendrimer", Journal of discrete mathematical sciences and cryptography, vol. 20, no. 2, pp. 515-523, Feb. 2017, doi: $10.1080 / 09720529.2016 .1220088$

[13] W. Gao and M. R. Farahani, "The Zagreb topological indices for a type of Benzenoid systems jagged-rectangle", Journal of interdisciplinary mathematics, vol. 20, no. 5, pp. 1341-1348, Jul. 2017, doi: $10.1080 / 09720502.2016 .1232037$

[14] W. Gao, L. Shi, and M. R. Farahani, "Szeged related indices of TUAC6[p, q]", Journal of discrete mathematical sciences and cryptography, vol. 20, no. 2, pp. 553-563, Feb. 2017, doi: $10.1080 / 09720529.2016 .1228312$

[15] W. Gao, M. Younas, A. Farooq, A. Virk, and W. Nazeer, "Some reverse degree-based topological indices and polynomials of dendrimers", Mathematics, vol. 6, no. 10, Art ID. 214, Oct. 2018, doi: $10.3390 /$ math 6100214

[16] I. Gutman, S. Klavzar, M. Petkovsekand and P. Zigert. "On Hosoya polynomials of benzenoid graphs", MATCH communication in mathematical and in computer chemistry, vol. 43, pp. 49-66, 2001. [On line]. Available: https://bitly/312kj74

[17] H. Hosoya, "On some counting polynomials in chemistry", Discrete applied mathematics, vol. 19 , no. 1-3, pp. 239-257, 1988, doi: 10.1016/0166-218X(88)90017-0 
[18] S. Klavžar and I. Gutman, "A comparison of the Schultz molecular topological index with the Wiener Index", Journal of chemical information and computer sciences, vol. 36, no. 5, pp. 1001-1003, Jan. 1996, doi: $10.1021 /$ ci9603689

[19] J.-B. Liu, M. Younas, M. Habib, M. Yousaf, and W. Nazeer, "M-polynomials and degree-based topological indices of $\mathrm{VC}_{5} \mathrm{C}_{7}[\mathrm{p}, \mathrm{q}]$ and $\mathrm{HC}_{5} \mathrm{C}_{7}[\mathrm{p}, \mathrm{q}]$ nanotubes", IEEE access, vol. 7, pp. 41125-41132, 2019, doi: 10.1109/ACCESS.2019.2907667

[20] B. Mohar and T. Pisanski, "How to compute the Wiener index of a graph", Journal of mathematical chemistry, vol. 2, no. 3, pp. 267-277, Jun. 1988, doi: 10.1007/BF01167206

[21] M. Munir, W. Nazeer, S. Rafique, and S. Kang, "M-polynomial and degree-based topological indices of polyhex nanotubes", Symmetry, vol. 8, no. 12, Art ID. 149, Dec. 2016, doi: 10.3390/sym8120149

[22] H. P. Schultz, "Topological organic chemistry. 1. Graph theory and topological indices of alkanes", Journal of chemical information and modeling, vol. 29, no. 3, pp. 227-228, Aug. 1989, doi: 10.1021/ci00063a012

[23] A. Shah and S. A. U. H. Bokhary, "On chromatic polynomial of certain families of dendrimer graphs", Open Journal of Mathematical Sciences, vol. 3, no. 1, pp. 404-416, Dec. 2019, doi: 10.30538/oms2019.0083

[24] V. Sharma, R. Goswami, and A. K. Madan, "Eccentric connectivity index: A novel highly discriminating topological descriptor for structureproperty and structure-activity studies", Journal of chemical information and computer sciences, vol. 37, no. 2, pp. 273-282, 1997, doi: $10.1021 / \mathrm{ci} 960049 \mathrm{~h}$

[25] H. M. A. Siddiqui and M. Imran, "Computing the metric dimension of wheel related graphs", Applied mathematics and computation, vol. 242, pp. 624-632, Sep. 2014, doi: 10.1016/j.amc.2014.06.006

[26] D. Stevanović, "Hosoya polynomial of composite graphs", Discrete mathematics, vol. 235, no. 1-3, pp. 237-244, May 2001, doi: 10.1016/S0012365X(00)00277-6

[27] A. Tabassum, M. A. Umar, M. Perveen, and A. Raheem, "Antimagicness of subdivided fans", Open journal of mathematical sciences, vol. 4, no. 1, pp. 18-22, Feb. 2020, doi: 10.30538/oms2020.0089 
[28] I. Tomescu, I. Javaid and I. Slamin, "On the partition dimension and connected partition dimension of wheels", Ars combinatoria, vol. 84, pp.311-318, 2007.

[29] T. Turaci, "The average lower 2-domination number of wheels related graphs and an algorithm", Mathematical and computational applications, vol. 21, Art ID. 29, Jul. 2016, doi: 10.20944/preprints201607.0037.v1

[30] M. A. Umar, N. Ali, A. Tabassum and B. R. Ali, "Book graphs are cycle antimagic", Open journal of mathematical sciences, vol. 3, no. 1, pp. 184-90, 2019, Jun. doi: 10.30538/oms2019.0061

[31] H. Wiener, "Structural determination of paraffin boiling points", Journal of the American Chemical Society, vol. 69, no. 1, pp. 17-20, Jan. 1947, doi: 10.1021/ja01193a005

[32] L. Yang, "Wiener index and traceable graphs", Bulletin of the Australian Mathematical Society, vol. 88, no. 3, pp. 380-383, Dec. 2013, doi: $10.1017 /$ S0004972712000901 